\title{
Fuzzy information graph of epileptic seizures
}

Tahir Ahmad and Vinod Ramachandran

Ibnu Sina Institute for Fundamental Science Studies, Universiti Teknologi Malaysia, 81310 Skudai Johor, MALAYSIA.

*Corresponding Author: tahir@ibnusina.utm.my (T. Ahmad)

Article history :

Received 11 March 2013

Revised 20 June 2013

Accepted 15 July 2013

Available online 2 August 2013

\section{GRAPHICAL ABSTRACT}

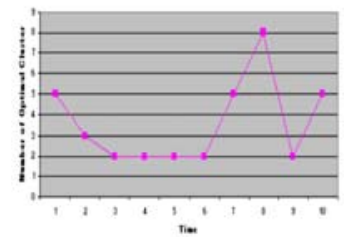

\section{ABSTRACT}

The mathematical modelling of EEG signals provides valuable data to neurologists, and is heavily utilized in the diagnosis and treatment of epilepsy. The erratic nature of these signals, coupled with their lack of a consistent visible trend results in a high degree of difficulty in forming a statistical model to describe seizures. Working with Delia-normalized signals, the authors compute the associated Shannon entropies for three sets of data, and show via construction that the information flow during an epileptic seizure can be viewed as a type-2 fuzzy graph.

Keywords: Electroencephalography, Epilepsy, Shannon entropy, Fuzzy graph

\section{INTRODUCTION}

Epilepsy is a chronic disorder of the brain that affects people all over the world. It is characterized by recurrent seizures - physical reactions to sudden, usually brief, excessive electrical discharges in a group of brain cells [1]. Seizures are classified into two major categories, partial seizures and generalized seizures. Partial seizures are those in which the clinical or electroencephalographic evidence suggests that the attacks have a localized onset in the brain [2]. This type involves only a part of the cerebral hemisphere at seizure onset and produces symptoms in corresponding parts of the body or disturbances in some related mental functions [3]. On the contrary, generalized seizures are said to occur if the evidence suggests that the attacks were well spread [4]. The diagnosis and treatment of epilepsy is greatly aided by the use of electroencephalography as a monitoring tool.

Electroencephalography (commonly referred to by its abbreviation EEG) is the measurement of electrical activity produced by the firing of neurons in the brain. It operates by recording the fluctuations in the potential difference of electrodes attached to the scalp of a patient, with these fluctuations indicating the presence of neural activity [5]. In practice, computerized systems such as NicoletOne are used to digitize EEG signals before they are subjected to statistical analysis. It is not uncommon for the interpretation of clinical EEG to involve speculation as to the possible locations of the sources inside the brain which are responsible for the observed activity on the scalp [4].

The mathematical analysis of EEG signals aids medical professionals by providing a description of the brain activity being observed, thus increasing the understanding of human brain activity.

\section{RELATED WORK}

To date, numerous methods have been employed in the mathematical modelling of epileptic seizures, each with varying objectives and results.

In 1986, Babloyantz and Destexhe used the time series of EEG data during a seizure to show the presence of deterministic dynamics of a complex nature for seizures [6]. By identifying and comparing the chaotic attractors during ictal and between interictal seizures, they argued that an epileptic seizure is a low-dimensional state, which was further clarified by Stam as a "loss of complexity" [7]. This concept was further strengthened by Iasemidis et al. by computing the decrease of the largest Lyapunov exponent during an epileptic seizure [8], a result which was further corroborated in 1990 by Frank's analysis of the EEG of absence seizures, in which he indicated the existence of an underlying chaotic attractor [9]. However, Theiler's analysis of the same dataset showed that the supposed chaotic properties can be attributed to a noisy limit cycle [10]. Support for this opposing view can be seen in studies by Schiff et al. [11], Friedrich and Uhl [12], Hernndez et al.[13], Le van Quyen et al. [14], and Feucht et al. [15], which leads to the conclusion that the use of time series modelling is highly dependent on the quality of the EEG signals produced. In practice, a noise filter is applied prior to signal amplification to help remove white noise. However, one inherent problem with EEG recordings of seizures is the crossing of its input and output sets - all recorded signals (gross output) are a 
combination of seizure activity (net output), exogenous noise, and the current source used to generate a potential difference between two electrodes (net input). Hence, to accurately model a seizure, dynamical noise reduction needs to be applied to isolate the signals generated from seizure activity alone, which in turn results in degradation of the source signal.

Nevertheless, modelling of the EEG signals of epileptic seizures endured, with focus shifting to forecasting brainstorms, reasoning for which is the ability to warn and prepare treatment for an impending seizure. The ultimate goal is to produce a closed system where a patient can be connected to an automated device that predicts the onset of seizures and administers the appropriate medication as necessary, a concept that was first outlined by Peters et al. in 2001 [16]. When it came to forecasting brainstorms, nonlinear models took preference over linear models. Studies conducted by Elger and Lehnertz [17], and Martinerie et al. used intercranial EEG to show that seizures can be anticipated, sometimes up to 5 minutes in advance [18]. In 2001, Le van Quyen demonstrated that seizure prediction can be carried out with surface recordings alone [7], jumpstarting research into noninvasive models for seizure prediction. Consequently, various approaches were employed to model seizures, including Lyapunov exponents [19] (shown to be inapplicable for low-dimensional deterministic chaotic systems such as epileptic seizures by Lai et al. In 2003 [20]), correlation integrals and dimensions [21] (also shown to be inapplicable for epileptic seizures [22]), phase clustering [8], [23], and entropy measures [24]. Although some of these models were quite successful, they are very specific in nature, only being able to model a very small subset of seizures a common flaw in nonlinear models. Additionally, Mormann et al. have highlighted that although most of the studies published in the 1990s and around the turn of the millennium yielded rather promising results, more recent evaluations could not reproduce these optimistic findings [25].

It was only after analysis of Martinerie's earlier work that McSharry et al. discovered that linear measures could also be used to model seizures [26]. The acceptability of linear models was solidified with Kugiumtzis and Larsson concluding that nonlinear methods

offered no significant advantage over linear models [27]. This result was followed up in 2001 by Jerger, who compared 7 different linear and nonlinear measures and found that both classes of measures produced similar results [28]. This provided strength to existing linear models, such as Baillet and Garnero's Bayesian-based model [29]. Although Bayesian modelling proved successful, its reliance on apriori information yields a low level of accuracy for patients who have a low occurrence of seizures. Furthermore, additional data cannot be generated by inducing seizures, as the Pavlovian effect on seizure provocation is still undetermined [30]; that is, researchers are unable to identify if repeated seizure provocation results in specific brain cells being conditioned to react to stimuli, thus jeopardizing the integrity of the data obtained.

With the introduction of new mathematical tools over the last decade, more complex models were developed. In 2008, Faust et al. successfully applied Burg autoregressive coefficients in the modelling of epileptic seizures, achieving an accuracy of over $90 \%$ in detecting seizures [31]. This result was bettered by Sivasankari and Thanushkodi in 2009 using fast independent component analysis coupled with neural networks [32]. These methods, although highly successful, are of high complexity and are notoriously resource intensive.

In 2000, Ahmad et al. formulated a fuzzy-based topological model to identify the foci of an epileptic seizure [33]. The model, called Fuzzy Topographic Topological Mapping (FTTM), involves inducing a topology on the magnetic field of magnetoencephalographic (MEG) recordings and utilizes fuzzy techniques to estimate the location of the epileptic foci [34]. The epileptic foci here refer to points in the brain from which the seizures are assumed to originate. This method was further refined in 2008 to include EEG input [3]. Apart from estimating the epileptic foci, the FTTM method is able to index key events during the progression of seizures, as demonstrated in Idris's 2010 paper [35].

In 2012, Ahmad and Ramachandran showed that by normalizing the raw EEG data of epileptic seizures allowed seizure data to be fitted to a unit hypersphere, allowing it to be modelled as a von-Mises Fisher (vMF) distribution [36]. Using the same patient data as Idris, the authors analyze the information content of the Delianormalized signals and compare them to the clustering results in [35]. Proof by construction is then used to show that the EEG signals of epileptic seizures can be used to generate a type-2 fuzzy graph.

\section{PRELIMINARIES}

The following definitions and/or theorems are used in this research:

Definition 1 (electrode set): The standard set of all monitored EEG electrodes during an epileptic seizure is called the electrode set, and is given by:

$$
E=\left\{e_{1}, e_{2}, \ldots, e_{n}\right\}
$$

where $e_{i}$ represents an EEG electrode that is being monitored during an epileptic seizure. 
Definition 2 (reference subset): The subset of the electrode set that contains only reference electrodes is called the reference subset, and is given by:

\section{$R=\{e \in E \mid e$ is a reference electrode $\}$.}

.Definition 3 (electrode potential set): In any given patient, the set of all potential differences detected during a seizure at time $t$ is given by:

$$
V_{t}=\left\{v_{e} \mid v_{e} \in \square, e \in E \backslash R\right\}
$$

where $V_{e}$ is the potential difference detected at electrode $e$ at time $t$ in relation to its closest reference electrode. $V_{t}$ is called the electrode potential set at time $t$.

Definition 4 (emulated baseline): The emulated baseline, $\beta$, is the average magnitude of electrical potentials detected across all monitored electrodes at a given time $t$ in a seizure, and is given by:

$$
\beta_{t}=\frac{\sum_{i=1}^{n}\left|v_{i}\right|}{n} \text { for } v_{i} \in V_{t}
$$

Definition 5 (jitter): The jitter, $\delta$, is the total difference in magnitude of detected electrical potentials and the emulated baseline at a given time in a seizure, and is given by:

$$
\delta_{t}=\sum_{i=1}^{n}|| v_{i}\left|-\beta_{t}\right| \text { for } v_{i} \in V_{t}
$$

Definition 6 (Delia EEG measure): The Delia EEG measure, $\mu$, is a measure defined on the power set of an electrode potential set, and is given by the set function: $\mu: P\left(V_{t}\right) \rightarrow \square_{e}$ such that

$$
\mu(A)= \begin{cases}0 & \text { if } n(A)=0, \\ \sum_{i=1}^{n}\left|\frac{\left|v_{i}\right|-\beta}{\delta}\right| & \text { otherwise }\end{cases}
$$

with the emulated baseline and jitter defined as in (3) and (4).

Definition 7 (surprisal): Surprisal, or self-information, is a measure of information content concerning the outcome of a random variable. It is given as

$$
I_{x}=-\log (p(x))
$$

Where $p(x)$ is the probability of outcome $x$ occurring. The unit of the surprisal measured depends on the base of the logarithm used, namely bits (base 2), nats (base), or dits (base 10) [37].

Definition 8 (Shannon entropy): Shannon entropy, $H$, or more commonly referred to as just entropy, is the expected value of the surprisal of a random variable, and is given in explicit form as

$$
H(X)=-\sum_{i=1}^{n} p\left(x_{i}\right) \log \left(p\left(x_{i}\right)\right)
$$

where $X$ is a random variable with $x \in X$ as an event. Simply put, entropy is the average information content that is missing from a random variable [37].

Theorem 1 (Shannon's source coding theorem): $N$ independent identically distributed random variables each with entropy $H(X)$ can be compressed into more than $N H(X)$ bits with negligible risk of information loss, as $N \rightarrow \infty$; but conversely, if they are compressed into fewer than $N H(X)$ bits it is virtually certain that information will be lost [37].

Definition 9 (fuzzy graph): A fuzzy graph is a graph in which the vertex and/or edge sets are fuzzy sets. For any graph $G(V, E)$, the functions $V_{G}$ and $E_{G}$ are defined such that $V_{G}: S \rightarrow[0,1], E_{G}: S \times S \rightarrow[0,1]$, and $E(x, y) \leq E(x) \wedge E(y)[38]$.

Theorem 2 (fuzzy graph taxonomy): There are five types of fuzzy graphs, namely:

1. Fuzzy set of graphs

2. Crisp vertex set in conjunction with a fuzzy edge set

3. Crisp vertex and edge sets in conjunction with fuzzy connectivity

4. Crisp edge set in conjunction with a fuzzy vertex set

5. Crisp graph with fuzzy weights [incineration].

\section{INFORMATIONAL CONTENT ANALYSIS}

A short, terminal-based program was written in $\mathrm{C}$ to automate the computation of the Delia measure, surprisal, and entropy values. Based on the values computed, three data sets were analyzed, namely a 10 -second focal seizure (Patient A), a 15-second generalized seizure (Patient B), 
and a 25-second focal seizure (Patient C). Seizure data is cropped to the period corresponding to ictal by our consulting neurologist, and is extracted to numerical form using Nicolet. Table 1 gives the entropy values of Patient A's seizure in one-second time blocks.

Due to the difference in length for each seizure, a direct comparison between them is impossible on a second-by-second basis (a comparison left to use of a vMF distribution). However, it is possible to compare their minimum and maximum entropies to formulate a hypothesis regarding the relation between seizure length and information content. This information is given in Table 2.

Based on the maximum amount of entropy at any given time $t$ during all three data sets, we can see that for the focal seizures, the overall entropy present decreases as the seizure duration increases. This indicates that the longer the ictal period of a focal seizure is, the more predictable it becomes.

Table 1 Entropic content of a 10-second seizure (Patient A).

\begin{tabular}{cc} 
Time, t (seconds) & Entropy, H (bits) \\
\hline 1 & 8463.0296 \\
2 & 7502.6510 \\
3 & 6906.1181 \\
4 & 4493.9316 \\
5 & 8070.0361 \\
6 & 11798.1141 \\
7 & 12937.2516 \\
8 & 14222.2185 \\
9 & 12578.9076 \\
10 & 8817.3858 \\
\hline
\end{tabular}

Table 2 Occurrence of minimum and maximum entropies for patients $\mathrm{A}, \mathrm{B}$, and $\mathrm{C}$.

\begin{tabular}{ccc}
\hline \multirow{2}{*}{ Patient } & \multicolumn{2}{c}{ Time of occurrence } \\
\cline { 2 - 3 } & Minimum, tmin (seconds) & Maximum, tmax (seconds) \\
\hline A & $4(4493.9316 \mathrm{H})$ & $8(14222.2185)$ \\
B & $3(6906.1181)$ & $5(17300.2411)$ \\
C & $21(2657.4386)$ & $2(9991.7922)$
\end{tabular}

It is also worth noting that a generalized seizure indicates a higher entropic content, even when compared to a focal seizure of greater length. This implies that focal seizures carry more information in comparison to generalized seizures. This difference in informational content is most likely corresponds to the additional spatial data carried by focal seizures that enables us to identify their point of action.

What's even more interesting is that the points of minimum/maximum entropy correspond to time frames of a seizure that optimally require the least/most clusters using Idris's non-polar EEG $C_{E E G}$ method, specifically at the $t=4 \mathrm{~s}$ and $t=8 \mathrm{~s}$ mark. This observation, in conjunction with Shannon's source coding theorem, indicates that epileptic seizures can be reconstructed from "building blocks", and the minimum number of clusters corresponds to the entropic lower bound of the information required to reconstruct the signal.

\section{GRAPH CONSTRUCTION}

Given the entropies at each time $t$, it is possible to create a graph of a seizure's progression in terms of information content.

Theorem 3 (fuzzy graph of epileptic seizures): The EEG signals during an epileptic seizure can be presented in the form of a type-2 fuzzy graph.

Proof:

(via construction)

Define the vertex set as follows:

$$
V_{G}=\{t \mid \text { an epileptic seizure is recorded at } t\}
$$

This results in a crisp vertex set.

Define the edge function to be:

$$
E_{G}=\left|\frac{H\left(x_{i}\right)-H\left(x_{j}\right)}{H\left(x_{i}\right)}\right|
$$

where $H\left(x_{i}\right)$ is the entropy at the vertex of edge origin, and $H\left(x_{j}\right)$ is the entropy at the vertex of edge termination Then, $G\left(V_{G}, E_{G}\right)$ is a fuzzy graph of type-2.

It can be seen from Figure 2 (b), generalized seizures gain/lose the bulk of their entropy during the beginning/ending stages of ictal. This observation however, is absent in focal seizures (Figure 2 (a) and Figure 2 (c)), where the rate of information gain/loss is somewhat distributed across ictal. This indicates that generalized seizures display a more aggressive change in brain activity in comparison to focal seizures, an observation that is not visible in standard EEG. 


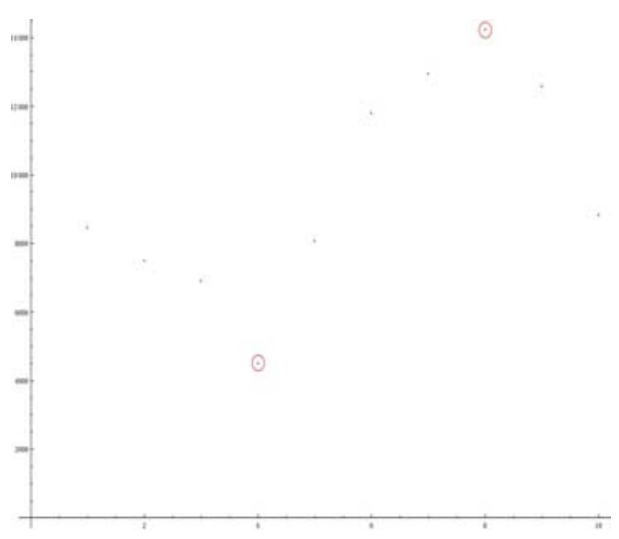

(a)

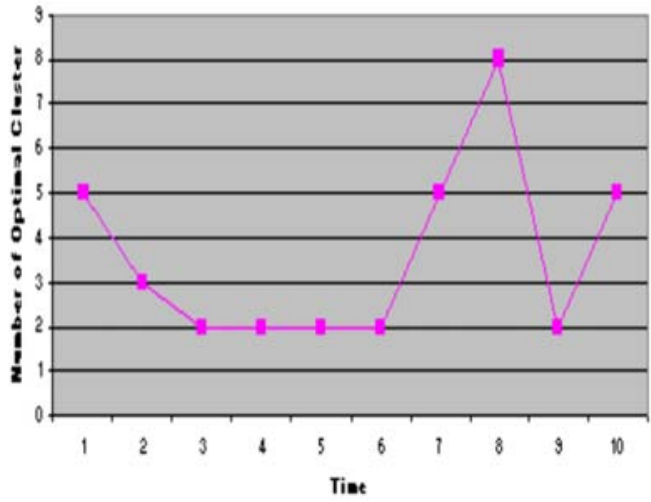

(b)

Figure 1 Comparison between entropic content and number of optimal clusters as computed by Idris. The minimum/maximum entropy circled in red (a) corresponds to points in (b) which optimally require the least/most number of clusters.

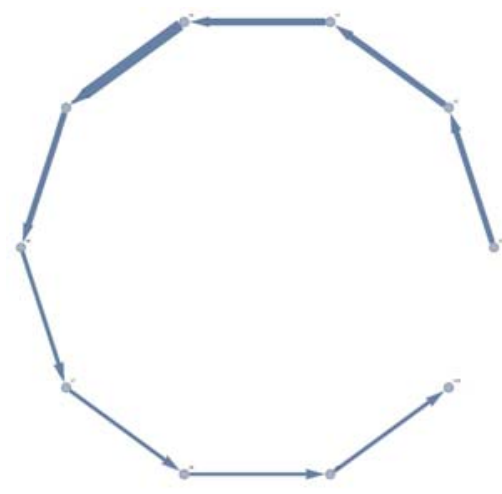

(a)

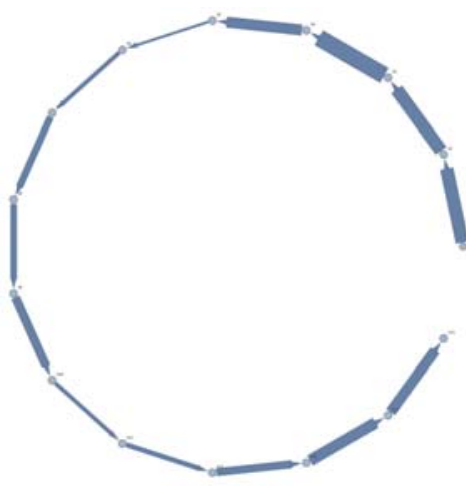

(b)

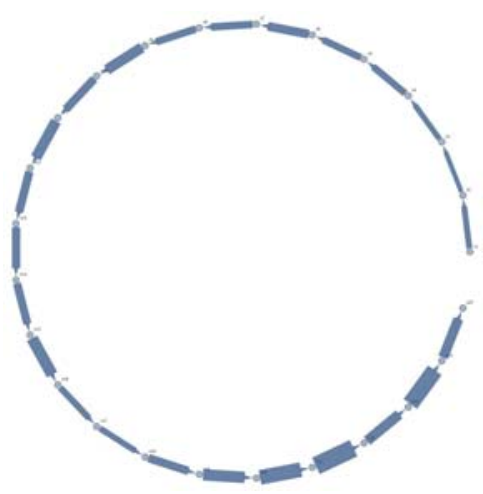

(c)

Figure 2 Fuzzy graphs for 10-second (a), 15-second (b), and 25-second (c) seizures. The edge thickness represents the amount of entropy lost/gained between the connected vertices.

\section{CONLCUSION}

In this paper, the entropic information of three epileptic seizures was computed. A comparison with the optimal number of clusters obtained via the $C_{E E G}$ method was made, and a correlation between the entropy and minimum cluster requirements were observed. A fuzzy graph of type- 2 was then constructed for each set of data, resulting in the observation of a more aggressive profile for generalized seizures in comparison to focal seizures.

\section{ACKNOWLEDGEMENT}

The authors would like to thank the Ministry of Higher Education for funding this research under the Fundamental Research Grant Scheme (Vot. No. 78315). One of the authors is also funded by the Ministry of Science, Technology and Innovation under the National Science Fellowship (NSF) grant.

\section{REFERENCES}

[1] WHO, Epilepsy factsheet, 2009.

[2] H. Gastaut, Epilepsia, vol. 11, no. 1, pp.102-112, 1970.

[3] T. Ahmad, R. A. Fairuz, F. Zakaria, and H. Isa,Proceedings of the 7th WSEAS InternationalConference on Signal Processing, Robotics and Automation, pp. 270-273, World Scientific and Engineering Academy and Society _WSEAS_,Stevens Point,Wis, USA, 2008.

[4] F. Zakaria, Universiti Teknologi Malaysia, 2008.

[5] E. Niedermeyer and F. H. L. Silva, Medicine Series, Lippincott Williams \&Wilkins, 2005.

[6] A. Babloyantz and A. Destexhe, Proceedings of the National Academy of Sciences, 83(10) (1986) 3513.

[7] C. J. Stam, Clinical Neurophysiology, 116(10) (2005) 2266.

[8] L. D. Iasemidis, S. J. Chris, H. P. Zaveri, and W. J. Williams, Brain Topography, 2(3)(1990) 187.

[9] G. W. Frank, T. Lookman, M. A. H. Nerenberg, C. Essex, J. Lemieux, and W. Blume, Physica D, 46(3)(1990) 427.

[10] J. Theiler, Physics Letters A, 196(5-6) (1995) 335.

[11] N. Schiff, J. Victor, A. Canel, and D. Labar, Biological Cybernetics, 72(6) (1995) 519.

[12] R. Friedrich and C. Uhl, Physica D, 98(1) (1996) 171. 
[13] J. L. Hernndez, P. A. Valds, and P. Vila, NeuroReport, 7(13) (1996) 2246

[14] M. Le van Quyen, J. Martinerie, C. Adam, and F. J. Varela, Physical Review E, 56(3) (1997) 3401.

[15] M. Feucht, U. Mller, H.Witte et al., Cerebral Cortex, 8(6) (1998) 524.

[16] T. E. Peters, N. C. Bhavaraju, M. G. Frei, and I. Osorio, Journal of Clinical Neurophysiology, 18(6) (2001) 545.

[17] C. E. Elger and K. Lehnertz, European Journal of Neuroscience, 10(2) (1998) 786.

[18] J. Martinerie, C. Adam, M. Le van Quyen et al., Nature Medicine, 4 (1998) 1173

[19] H. R. Moser, B. Weber, H. G. Wieser, and P. F. Meier, Physica D, 130(3-4) (1999) 291.

[20] Y. C. Lai, M. A. F. Harrison, M. G. Frei, and I. Osorio, Physical Review Letters, 91(6) (2003) Article ID 068102.

[21] I. Osorio, M. A. Harrison, Y. C. Lai, andM. G. Frei, Journal of Clinical Neurophysiology, 18(3) (2001) 269.

[22] M. A. F. Harrison, I. Osorio, M. G. Frei, S. Asuri, and Y. C. Lai, Chaos, 15(3) (2005) Article ID 33106.

[23] S. Kalitzin, J. Parra, D. N. Velis, and F. H. Lopes da Silva, IEEE Transactions on Biomedical Engineering, 49(11) (2002) 1279.

[24] W. van Drongelen, S. Nayak, D. M. Frim et al., Pediatric Neurology, 29(3) (2003) 207.

[25] F. Mormann, R. G. Andrzejak, C. E. Elger, and K. Lehnertz, Brain, 130(2) (2007) 314.

[26] P. McSharry, T. He, L. Smith, and L. Tarassenko, Medical and Biological Engineering and Computing, 40(4) (2002) 447.
[27] D. Kugiumtzis and P. G. Larsson, Chaos in Brain?: Proceedings of theWorkshop, K. Lehnertz and C. E. Elger, Eds., pp. 329-332, World Scientific, 1999.

[28] K. K. Jerger, T. I. Netoff, J. T. Francis et al., Journal of Clinical Neurophysiology, 18 (2001) 259.

[29] S. Baillet and L. Garnero, IEEE Transactions on Biomedical Engineering, 44(5) (1997) 374.

[30] J. R. StevensElectroencephalography and Clinical Neurophysiology, 12(2) (1960) 431.

[31] O. Faust, R. U. Acharya, A. R. Allen, and C. M. LinIng'enierie et Recherche Biom'edicale, 29(1) (2008) 44.

[32] N. Sivasankari and K. Thanushkodi, International Journal of Soft Computing Applications, 1(2) (2009) 1.

[33] T. Ahmad, R. S. Ahmad, F. Zakaria, and L. L. Yun, in Proceedings of the BIOMED 2000, pp. 119-121, University of Malaya, September 2000.

[34] T. Ahmad, R. S. Ahmad, W. E. Z. W. Abdul Rahman, L. L. Yun, and F. Zakaria, Journal of Interdisciplinary Mathematics, 11 (2008) 381.

[35] A. Idris, T. Ahmad, and N. Maanin Proceedings of the International Conference on Applied Mathematics and Informatics, pp. 94-99, 2010.

[36] Tahir Ahmad and Vinod Ramachandran, Journal of Applied Mathematics, (2012) Article ID 926358. doi:10.1155/2012/926358

[37] Thomas M. Cover, Joy A. Thomas, John Wiley \& Sons, 2006.

[38] Tahir, A., Sabariah, B., Arshad, Khairil, Journal of Mathematical Chemistry 47(4) (2010) 1263. 